\title{
Yang-Mills Propagators and QCD *
}

\author{
Marco Frasca \\ Via Erasmo Gattamelata, 3 \\ 00176 Roma (Italia)
}

We present a strong coupling expansion that permits to develop analysis of quantum field theory in the infrared limit. Application to a quartic massless scalar field gives a massive spectrum and the propagator in this regime. We extend the approach to a pure Yang-Mills theory obtaining analogous results. The gluon propagator is compared satisfactorily with lattice results and similarly for the spectrum. Comparison with experimental low energy spectrum of QCD supports the view that $\sigma$ resonance is indeed , a glueball. The gluon propagator we obtained is finally used to formulate a low energy Lagrangian for QCD that reduces to a Nambu-Jona-Lasinio model with all the parameters fixed by those of the full theory.

One of the main difficulties people meets to cope with low energy limit of a quantum field theory is the missing of ' a perturbation techniques that could permit to extract analytical manageable results to compare with experiment. In the fall of seventies, Bender's group proposed a possible method to solve this impasse 1/234. Bender's group ap'proach can be understood with a simple quartic oscillator as (here and following $\hbar=c=1$ )

$G\left(t_{2}-t_{1}\right)=\int[d q(t)] e^{i \int_{t_{1}}^{t_{2}} d t\left[\frac{1}{2} \dot{q}(t)^{2}-\frac{\lambda}{4} q(t)^{4}\right]}$

When the limit $\lambda \rightarrow \infty$ is taken one simply neglects the kinematic term assuming it as a higher order correction leaving us with

$G\left(t_{2}-t_{1}\right) \approx \int[d q(t)] e^{-i \int_{t_{1}}^{t_{2}} d t \frac{\lambda}{4} q(t)^{4}}$

a highly singular expression that needs to be regularized ' with a proper cut-off. This in turn implies that computations produce series generally difficult to resum due to the very singular dependence on the cut-off, and the method proves to be not effective in the computation of propagators and spectra. The reason why Bender's group method does not work properly is due to the completely omitted dynamics that should be permitted instead. This can be accomplished if we do a time rescaling as $t \rightarrow \sqrt{\lambda} t=\tau$ and we are left with the expression

$G\left(\tau_{2}-\tau_{1}\right)=\int[d q(\tau)] e^{i \sqrt{\lambda} \int_{\tau_{1}}^{\tau_{2}} d \tau\left[\frac{1}{2} \dot{q}(\tau)^{2}-\frac{1}{4} q(\tau)^{4}\right]}$

that is perfectly regular being just the semiclassical limit when $\lambda \rightarrow \infty$. In this case the spectrum is computed straightforwardly and, accounting for all higher order corrections, one has the exact result [5]. Starting from the Schrödinger equation

$$
-\frac{\hbar^{2}}{2 m} \frac{\partial^{2} \psi}{\partial x^{2}}+\frac{\lambda}{4} x^{4} \psi=i \hbar \frac{\partial \psi}{\partial t},
$$

rescaling time as $\tau=\sqrt{\lambda} t$ one has

$-\frac{1}{\lambda} \frac{\hbar^{2}}{2 m} \frac{\partial^{2} \psi}{\partial x^{2}}+\frac{1}{4} x^{4} \psi=i \hbar \frac{\partial \psi}{\partial \tau}$

*Talk given at QCD 08 International Conference (Montpellier, 7-12th July 2008) and finally taking an expansion like $\psi=\psi^{(0)}+\frac{1}{\lambda} \psi^{(1)}+$ $\frac{1}{\lambda^{2}} \psi^{(2)}+O\left(\frac{1}{\lambda^{3}}\right)$ for the wave function, the above method recovers a well-known semiclassical expansion,

$$
\begin{aligned}
\psi= & e^{-\frac{i}{\hbar} \frac{x^{4}}{4} \tau}\left\{\phi(x)-\frac{\hbar^{2}}{2 m}\left[\frac{\partial^{2} \phi(x)}{\partial x^{2}}\right.\right. \\
& \left.\left.-2 \frac{i}{\hbar} x^{3} \tau \frac{\partial \phi(x)}{\partial x}-\left(3 \frac{i}{\hbar} x^{2} \tau+\frac{1}{\hbar^{2}} x^{6} \tau^{2}\right) \phi(x)\right]+\ldots\right\}
\end{aligned}
$$

that is the Wigner-Kirkwood expansion with the spectrum given by th usual WKB series [6]. But Wigner-Kirkwood expansion is a gradient expansion and this is the key result: A strongly coupled quantum system behaves semiclassically and its behavior is described by a Wigner-Kirkwood gradient expansion. A typical application for this fundamental result could be measurement theory when a measuring apparatus is strongly coupled to a quantum system. But the method is quite general to be applicable to whatever differential equation.

We would like to extend the above approach to quantum field theory. For our aims, we consider a massless quartic scalar field theory with a generating functional

$Z[j]=\int[d \phi] e^{i \int d^{4} x\left[\frac{1}{2}(\partial \phi)^{2}-\frac{\lambda}{4} \phi^{4}+j \phi\right]}$

and rescale time as shown above for the quartic oscillator to obtain

$Z[j]=\int[d \phi] e^{i \sqrt{\lambda} \int d^{4} x\left[\frac{1}{2}(\dot{\phi})^{2}-\frac{1}{4} \phi^{4}+j \phi\right]} e^{-\frac{i}{\sqrt{\lambda}} \int d^{4} x \frac{1}{2}(\nabla \phi)^{2}}$

that in the limit $\lambda \rightarrow \infty$ permits us to recover again the semiclassical limit and a gradient expansion [7. But in this case we are able to solve the equation for the propagator

$\ddot{G}\left(t_{1}-t_{2}\right)+\lambda G\left(t_{1}-t_{2}\right)^{3}=\mu^{2} \delta\left(t_{1}-t_{2}\right)$

that is

$G\left(t_{1}-t_{2}\right)=\theta\left(t_{1}-t_{2}\right) \mu\left(\frac{2}{\lambda}\right)^{\frac{1}{4}} \mathrm{sn}\left[\left(\frac{\lambda}{2}\right)^{\frac{1}{4}} \mu t\right]$

being sn a Jacobi elliptic function and $\mu$ an arbitrary integration constant. Now, using a small time approximation we can take the following definition for the field $\phi$ [89]

$\phi(t) \approx \int d t^{\prime} G\left(t-t^{\prime}\right) j\left(t^{\prime}\right)$ 
we can restate the above generating functional for the scalar field theory in a Gaussian form

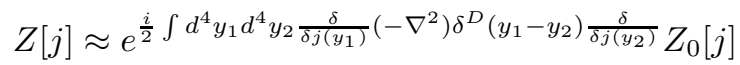

being

$Z_{0}[j]=e^{\frac{i}{2} \int d^{4} x_{1} d^{4} x_{2} j\left(x_{1}\right) \Delta\left(x_{1}-x_{2}\right) j\left(x_{2}\right)}$

with $\Delta\left(x_{1}-x_{2}\right)=\delta^{3}\left(x_{1}-x_{2}\right)\left[\theta\left(t_{1}-t_{2}\right) G\left(t_{1}-t_{2}\right)+\theta\left(t_{2}-\right.\right.$ $\left.\left.t_{1}\right) G\left(t_{2}-t_{1}\right)\right]$. Having obtained the propagator in the strong coupling limit we can extract the spectrum of the theory in the infrared limit. This can be done using eq.(10) and the relation for the sn Jacobi function 10

$\operatorname{sn}(u, i)=\frac{2 \pi}{K(i)} \sum_{n=0}^{\infty} \frac{(-1)^{n} e^{-\left(n+\frac{1}{2}\right) \pi}}{1+e^{-(2 n+1) \pi}} \sin \left[(2 n+1) \frac{\pi u}{2 K(i)}\right]$

being $K(i)=\int_{0}^{\frac{\pi}{2}} \frac{d \theta}{\sqrt{1+\sin ^{2} \theta}} \approx 1.3111028777$. This gives in our case for the Feynman propagator

$\Delta(\omega)=\sum_{n=0}^{\infty}(2 n+1) \frac{\pi^{2}}{K^{2}(i)} \frac{(-1)^{n+1} e^{-\left(n+\frac{1}{2}\right) \pi}}{1+e^{-(2 n+1) \pi}} \frac{1}{\omega^{2}-\omega_{n}^{2}+i \epsilon}$

with the harmonic oscillator spectrum

$\omega_{n}=\left(n+\frac{1}{2}\right) \frac{\pi}{K(i)}\left(\frac{\lambda}{2}\right)^{\frac{1}{4}} \mu$.

We see immediately that the propagator is in agreement with Källen-Lehman representation. This is a key observation if we want to interpret the poles of the propagator as physical states even if the propagator is gauge dependent. But we also see that this propagator satisfies the Callan-Symanzik equation

$\mu \frac{\partial G(t)}{\partial \mu}+\beta(\lambda) \frac{\partial G(t)}{\partial \lambda}+2 \gamma G(t)=0$

with a beta function $\beta=-4 \lambda$ and $\gamma=-1$. This means that the theory is trivial in the infrared limit as the coupling goes to zero as the fourth power of momentum [11.

The next step is to apply the above approach to a pure Yang-Mills theory in order to analyze the behavior in the low energy limit. But we have to cope here with a serious difficulty. In the eighties it was proved by Matinyan, Savvidy, et al. 12 13 14 that the classical equations of motion of Yang-Mills theory are generally chaotic and so, completely useless to built a quantum field theory. The way out is the following mapping theorem [15]:

MAPPING THEOREM: An extremum of the action

$S=\int d^{4} x\left[\frac{1}{2}(\partial \phi)^{2}-\frac{\lambda}{4} \phi^{4}\right]$

is also an extremum of the $S U(N)$ Yang-Mills Lagrangian when we properly choose $A_{\mu}^{a}$ with some components being zero and all others being equal, and $\lambda=N g^{2}$, being $g$ the coupling constant of the Yang-Mills field.

This theorem holds exactly at classical level but at a quantum level can be maintained only in the infrared limit as in the ultraviolet case quantum fluctuations spoil the mapping producing asymptotic freedom, a property missing for the scalar field [16]. Then, using mapping theorem we can write down immediately the gluon propagator in the Landau gauge as

$$
\begin{aligned}
D_{\mu \nu}^{a b}(p)= & \delta^{a b}\left(\eta_{\mu \nu}-\frac{p_{\mu} p_{\nu}}{p^{2}}\right) \sum_{n=0}^{\infty}(2 n+1) \frac{\pi^{2}}{K^{2}(i)} \times \\
& \frac{(-1)^{n+1} e^{-\left(n+\frac{1}{2}\right) \pi}}{1+e^{-(2 n+1) \pi}} \frac{1}{p^{2}-m_{n}^{2}+i \epsilon}
\end{aligned}
$$

where we have also introduced Latin indexes for color degree of freedom and

$m_{n}=\left(n+\frac{1}{2}\right) \frac{\pi}{K(i)}\left(\frac{N g^{2}}{2}\right)^{\frac{1}{4}} \Lambda$.

being $\Lambda$ the integration constant. Mapping theorem tells us also that the ghost field decouples from the gluon field and must behave like a free particle with a propagator $G(p)=1 /\left(p^{2}+i \epsilon\right)$. The running coupling must go to zero like the fourth power of momentum being this in agreement with lattice computations as shown by Boucaud et al. 17 but as also seen from experiments as shown by Prosperi et al. 181920]. These latter results on the running coupling give a strong clue that Yang-Mills theory is trivial as happens for the scalar field theory. Lattice computations give also results on the propagators $21,22,23$. These show that the ghost is very near the free particle case while the gluon propagator reaches a finite value at zero momentum. So, we compare our propagator (19) with lattice computations taking the one with the largest volume $(27 \mathrm{fm})^{4}[22$. For this to work we need to go to Euclidean momenta and fix $m_{0}$ in eq.(20). The result is presented in Fig. 1 and is really satisfactory.

The comparison is done in our case with $m_{0}=$ $\frac{\pi}{2 K(i)}\left(N g^{2} / 2\right)^{\frac{1}{4}} \Lambda \approx 547 \mathrm{MeV}$ corresponding to a string tension $\sqrt{\sigma}=\left(N g^{2} / 2\right)^{\frac{1}{4}} \Lambda \approx 456 \mathrm{MeV}$. This result is striking indeed as we observe a mass for the ground state very near that of the $\sigma$ resonance and a string tension very near that generally obtained from experiments $410-440 \pm 20 \mathrm{MeV}$. So, on the lattice the scenario is clearly settled. Numerical solution of Dyson-Schwinger equations also confirm it 24 .

For the spectrum the situation is satisfactory as well. Comparing with lattice computations by Teper et al. 25. 26], we have shown in 27] that one gets Tab.1 and 2. Theoretical numbers compared in the tables are computed through the relation $\frac{m_{n}}{m_{0}}=(2 n+1) \frac{\pi}{2 K(i)}$ that defines a set of "golden numbers" that are those measured in lattice computations of spectra.

We cannot say the same with the data by C. Morningstar et al. 28] but these authors use anisotropic lattices. The $\sigma$ state appears on lattice computations of the gluon propagator but not on lattice computations of spectra. It should be said that presently the volumes used in the former computations are far larger than for the latter 


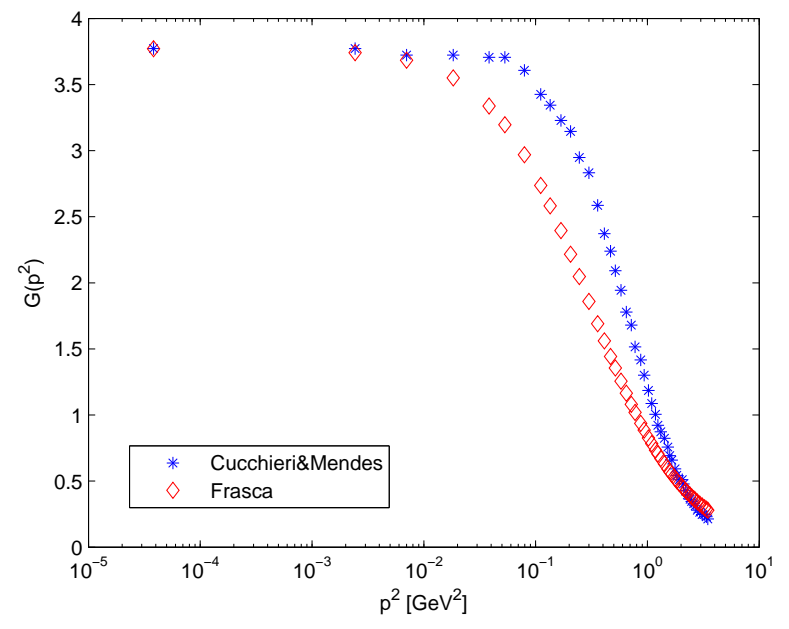

Figure 1. Fit to lattice data of propagator in eq. (19).

\begin{tabular}{|c|c|c|c|}
\hline \hline Excitation & Lattice & Theoretical & Error \\
\hline$\sigma$ & - & 1.198140235 & - \\
\hline $0^{++}$ & $3.55(7)$ & 3.594420705 & $1 \%$ \\
\hline $0^{++*}$ & $5.69(10)$ & 5.990701175 & $5 \%$ \\
\hline \hline
\end{tabular}

Table 1

Comparison for the $0^{++}$glueball

\begin{tabular}{|c|c|c|c|}
\hline \hline Excitation & Lattice & Theoretical & Error \\
\hline$\sigma^{*}$ & - & 2.396280470 & - \\
\hline $2^{++}$ & $4.78(9)$ & 4.792560940 & $0.2 \%$ \\
\hline $2^{++*}$ & - & 7.188841410 & - \\
\hline \hline
\end{tabular}

Table 2

Comparison for the $2^{++}$glueball case. Our view is that an effort should be done in this direction in order to get a clear understanding of YangMills theory on the lattice by increasing the volumes for spectrum computation.

A needed step is to understand current phenomenology of the low energy spectrum of QCD with the above results. Indeed, the ground state of a pure Yang-Mills theory can be identified with the observed resonance $\sigma$ or $f_{0}(600)$. This means that Yang-Mills theory has a higher ground state than full QCD as quarks lower it to the pion mass. Indeed, $f_{0}(600)$ or $\sigma$ seems to have a large gluonic content due to the very small width for $\gamma \gamma$ decay that can be due to $\pi \pi$ rescattering 29130 and as also seen through $\chi \mathrm{PT}$ 3132. We have shown that our computed mass is very near the one obtained from experiments [33. If we take for the string tension $440 \mathrm{MeV}$ as is customary in lattice computations we get $m_{\sigma}=527 \mathrm{MeV}$ and, as seen above, lattice computations for the gluon propagator give about $547 \mathrm{MeV}$. Mixing with quarks could reduce this value. A more conservative choice of $410 \mathrm{MeV}$ can take both $\sigma$ and $\sigma^{*}$, the latter eventually being $f_{0}(980)$, to agree with measured data giving $m_{\sigma} \approx 491 \mathrm{MeV}$ and $m_{\sigma^{*}} \approx 982 \mathrm{MeV}$. This view is shared in recent analysis 29130. But while these resonances are seen both experimentally and theoretically and, in indirect way through lattice computations for the propagator, a direct evidence on lattice computations for spectra appears difficult to achieve presently [34].

As pointed out in [35, once gluon propagator is known one can obtain a low energy model directly from QCD. We derived this model in [36. So, taking QCD action as

$$
\begin{aligned}
S= & \int d^{4} x\left[\sum_{q} \bar{q}\left(i \gamma \cdot \partial-g \frac{\lambda^{a}}{2} \gamma \cdot A^{a}-m_{q}\right) q\right. \\
& \left.-\frac{1}{4} G_{\mu \nu}^{a} G^{a \mu \nu}-\frac{1}{2 \alpha}(\partial \cdot A)^{2}\right]
\end{aligned}
$$

being $G_{\mu \nu}^{a}=\partial_{\mu} A_{\nu}^{a}-\partial_{\nu} A_{\mu}^{a}+g f^{a b c} A_{\mu}^{b} A_{\nu}^{c}$, we use the small time approximation seen for the quartic scalar and YangMills fields [89] with the above computed propagator writing

$A_{\mu}^{a}(x) \approx g \int d^{4} y D_{\mu \nu}^{a b}(x-y) \sum_{q} \bar{q}(y) \frac{\lambda^{a}}{2} \gamma^{\nu} q(y)$

giving

$$
\begin{aligned}
S \approx & \int d^{4} x\left[\sum_{q} \bar{q}(x)\left(i \gamma \cdot \partial-m_{q}\right) q(x)\right. \\
& -\frac{1}{2} g^{2} \sum_{q, q^{\prime}} \bar{q}(x) \frac{\lambda^{a}}{2} \gamma^{\mu} q(x) \times \\
& \left.\int d^{4} y D_{\mu \nu}^{a b}(x-y) \bar{q}^{\prime}(y) \frac{\lambda^{b}}{2} \gamma^{\nu} q^{\prime}(y)\right] .
\end{aligned}
$$

In the infrared limit $p \ll m_{0}$, being $m_{0}=$ $\left(N g^{2} / 2\right)^{\frac{1}{4}} \pi / 2 K(i) \Lambda=m_{\sigma} \approx 491 M e V$ that can be taken 
as the NJL cut-off $\Lambda_{N J L}$, one gets

$$
\begin{aligned}
S \approx & \int d^{4} x\left[\sum_{q} \bar{q}(x)\left(i \gamma \cdot \partial-m_{q}\right) q(x)\right. \\
& -\frac{1}{2} g^{2} \sum_{q, q^{\prime}} \bar{q}(x) \frac{\lambda^{a}}{2} \gamma^{\mu} q(x) \times \\
& \left.\int d^{4} y G(x-y) \bar{q}^{\prime}(y) \frac{\lambda^{a}}{2} \gamma_{\mu} q^{\prime}(y)\right] .
\end{aligned}
$$

being $G(x-y) \approx 3.761402959 \cdot \frac{1}{\sigma} \delta^{4}(x-y)$ (Fermi approximation). This gives in the end

$$
\begin{aligned}
S \approx & \int d^{4} x\left[\sum_{q} \bar{q}(x)\left(i \gamma \cdot \partial-m_{q}\right) q(x)\right. \\
& \left.-\frac{1}{2} G_{N J L} \sum_{q, q^{\prime}} \bar{q}(x) \frac{\lambda^{a}}{2} \gamma^{\mu} q(x) \bar{q}^{\prime}(x) \frac{\lambda^{a}}{2} \gamma_{\mu} q^{\prime}(x)\right]
\end{aligned}
$$

being $G_{N J L} \approx 3.761402959 \cdot \frac{g^{2}}{\sigma}$ that links QCD theory parameters and the coupling of the NJL model. The point opened up by the introduction of quarks is how to recover gluonic contributions to QCD spectrum. This possibility can be achieved through bosonization techniques 37 .

So, a consistent formulation of quantum field theory in the infrared exists. We have seen that a satisfactory agreement can be obtained in this way with lattice computations both for the propagators and the spectrum for YangMills theory. But while for the propagators the agreement is really good, for the spectrum some more effort on lattice computations seems needed to obtain a satisfactory comprehension of all the matter. From the experimental side the situation is quite good granting an understanding of where glueballs should lie in the observed spectrum. Agreement is seen between different theoretical approaches in this case while some more work is needed to clarify the situation. It should be said that if further elements will confirm this scenario, surely a lot of unexpected views about strong interactions will force new understanding for all quantum field theory.

\section{REFERENCES}

1. C. M. Bender, F. Cooper, G. S. Guralnik, and D. H. Sharp, Phys. Rev. D 19, (1979) 1865.

2. C. M. Bender, F. Cooper, G. S. Guralnik, D. H. Sharp, R. Roskies, and M. L. Silverstein, Phys. Rev. D 20, (1979) 1374 .

3. C. M. Bender, F. Cooper, G. S. Guralnik, H. Moreno, R. Roskies, and D. H. Sharp, Phys. Rev. Lett. 45, (1980) 501 .

4. C. M. Bender, F. Cooper, R. Kenway, and L. M. Simmons, Phys. Rev. D 24, (1981) 2693.

5. C. M. Bender, K. Olaussen and P. S. Wang, Phys. Rev. D 16, (1977) 1740 .

6. M. Frasca, Proc. R. Soc. A (2007) 463, 2195.

7. M. Frasca, Phys. Rev. D 73, (2006) 027701 ; Erratumibid., (2006) 049902.
8. M. Frasca, Mod. Phys. Lett. A 22, (2007) 1293.

9. M. Frasca, Int. J. Mod. Phys. A 23 (2008) 299.

10. I. S. Gradshteyn, I. M. Ryzhik, Table of Integrals, Series, and Products, (Academic Press, 2000).

11. M. Frasca, arXiv:0802.1183 [hep-th].

12. S. G. Matinyan, G. K. Savvidy, N. G. Ter-Arutunian Savvidy, Sov. Phys. JETP 53, 421 (1981).

13. G. K. Savvidy, Phys. Lett. B 130, 303 (1983).

14. G. K. Savvidy, Nucl. Phys. B 246, 302 (1984).

15. M. Frasca, arXiv:0709.2042 [hep-th].

16. M. E. Peskin, D. V. Schroeder, An Introduction to Quantum Field Theory, (Perseus, Reading, 1995), Ch.12.

17. Ph. Boucaud, F. De Soto, A. Le Yaouanc, J.P. Leroy, J. Micheli, H. Moutarde, O. Pène, J. Rodrguez-Quintero, JHEP 0304 (2003) 005.

18. G. M. Prosperi, M. Raciti, C. Simolo, Prog. Part. Nucl. Phys. 58, 387 (2007).

19. M. Baldicchi, A. V. Nesterenko, G. M. Prosperi, D. V. Shirkov, C. Simolo, Phys. Rev. Lett. 99, 242001 (2007).

20. M. Baldicchi, A. V. Nesterenko, G. M. Prosperi, C. Simolo, arXiv:0705.1695 [hep-ph].

21. I.L. Bogolubsky, V.G. Bornyakov, G. Burgio, E.M. Ilgenfritz, V.K. Mitrjushkin, M. Müller-Preussker, P. Schemel, PoS (LATTICE 2007) 318.

22. A. Cucchieri, T. Mendes, PoS (LATTICE 2007) 297.

23. A. Sternbeck, L. von Smekal, D. B. Leinweber, A. G. Williams, PoS (LATTICE 2007) 340.

24. A. C. Aguilar, A. A. Natale, JHEP 0408 (2004) 057.

25. B. Lucini, M. Teper, Phys. Rev. D 64, 105019 (2001).

26. B. Lucini, M. Teper, U. Wenger, JHEP 06, 012 (2004).

27. M. Frasca, arXiv:0704.3260 [hep-th].

28. Y. Chen, A. Alexandru, S. J. Dong, T. Draper, I. Horvath, F. X. Lee, K. F. Liu, N. Mathur, C. Morningstar, M. Peardon, S. Tamhankar, B. L. Young, J. B. Zhang, Phys. Rev. D 73, 014516 (2006).

29. G. Mennessier, S. Narison, W. Ochs, 0804.4452 [hep$\mathrm{ph}$.

30. G. Mennessier, P. Minkowski, S. Narison, W. Ochs, 0707.4511 [hep-ph].

31. J. R. Pelaez, G. Rios, Phys. Rev. Lett. 97 (2006) 242002.

32. C. Hanhart, J. R. Pelaez, G.Rios, 0801.2871 [hep-ph].

33. D.V. Bugg, 0804.3450 [hep-ph].

34. C. McNeile, 0710.2470 [hep-lat].

35. T. Goldman, R. W. Haymaker, Phys. Rev. D 24, 724 (1981).

36. M. Frasca, arXiv:0803.0321 [hep-th].

37. D. Ebert, hep-ph/9710511 